\begin{tabular}{ccc}
\hline & International Journal of Engineering \& Technology, $7(2.7)(2018) 266-269$ \\
SPC & International Journal of Engineering \& Technology \\
Website: $w$ ww.sciencepubco.com/index.php/IJET & Research Paper \\
\hline
\end{tabular}

\title{
Mobile based home mechanization framework using IoT for smart cities
}

\author{
Dr. K. V. Daya Sagar ${ }^{1}$ *, P Sai Durga ${ }^{2}$, G. Kavya ${ }^{2}$, K Sri Sravya ${ }^{2}$, K. Krishna Veni ${ }^{1}$ \\ ${ }^{1}$ Associate Professor, Department of Electronics and Computer Science Engineering, KLEF Deemed to be University \\ ${ }^{2}$ Dept. of Computer Science \& Engineering, KLEF Deemed to be University \\ *Corresponding author E-mail: saidurgareddy4121@gmail.com
}

\begin{abstract}
A web of things abstracts the likelihood of distantly interfacing and detecting correct questions (things) through the web. With regards to our house, this knowledge can be apropos consolidated to brand it more intelligent, extra secured and robotization IoT venture focuses on constructing a remote home safety framework which directs caution to the user by using web if there is an incidence of any intruder it raises an alert automatically. Moreover, the identical can be similarly utilized for the home automation by using an analogous organization of devices. The use developed by desiring this context over the relative kinds of prevailing settings is that alarm and the position sent by the Wi-Fi linked microcontroller oversaw context can be gotten by the client on the telephone on or after in the least separation regardless of his adaptable telephone is linked with the web or not. The microcontroller used as a present model is the TICC 3220 Launchpad, which attends a fixed smaller measure regulator besides a nearby available Wi-Fi making use of all the electrical machinery inside the house can be measured and supervised.
\end{abstract}

Keywords: Microcontroller; WIFI; Launch Pad; TICC3220; MSP435; Embedded Systems; Internet of Things.

\section{Introduction}

Distant home Safety and household mechanization stand the dual part of this task. The as of now invented faultless of the context directs an alarm to the administrator over speech calls by means of the web. Uncertainty any kind of humanoid expansion is spotted close to the channel of his house then raises an alert upon the customer's caution. The preparation for transfer prepared messages to troubled refuge staff if nearby ought to be an incidence of the elementary condition is furthermore merged into the agenda. Then again if the administrator distinguishes that the distinct going into his house isn't a gate crasher, however, a surprise guest of his, then as different to situation tainted safekeeping caution, the customer can make bases of act such as introducing the entrance , swapping on diverse machineries private the home, which is furthermore related and measured by the trivial rule organizer in the outline on the way to comfy his guest, the similar should be probable when the customer himself drives into the area, he can make himself at full ease deprived of actually converting on the electrical devices or television channel for a photograph

In this way, using sensors, the dual issues of house security and home robotization can be commenced on a cooperation premise. The alarms and rank of the IoT outline remain acquired through customer as of any place wherever web access might not stand promptly available. The present infra-red or Bluetooth distant panels exhibit cutting-edge the bazaar is when all is said in done machine precise then the similar can't be used equally. Electrical gadgets associated with Bluetooth authorized cell phones can't be supervised from a detached area. Along these lines measurements, for example, having the capacity to turn a ventilation and cooling system. While returning home is impossible with such backgrounds.In distinguish, this work gives a financial practicality and straightforward preparation for remote house mechanization and security home framework. The distress looked by present home security frameworks in giving data connecting to the circumstances to the customer when being away from home to get rid of this situation .the ensuing parts of the paper have been collected after a relative examination amid the anticipated framework plus current sector

\section{Setup components}

1) TI CC3220S launch-pad.

2) WI-FI router.

3) Camera-MT9D111.

4) Android Mobile App.

5) Alarm as an observant.

6) Temperature LM35 Sensor

7) LCD Display

\subsection{TI CC3220S Launchpad}

The TI CC3220s is a Simple Link ${ }^{\mathrm{TM}}$ microcontroller (MCU) consisting of Wi-Fi, truncated imperativeness, Sub-1 GHz and hosts MCUs, which gives a regular, easy way to-utilize and change condition by a middle programming propelled unit (SDK) and rich gadget set.

The SimpleLink ${ }^{\mathrm{TM}}$ CC3220S gadget family is a solitary chip arrangement, joining two bodily isolated, on-chip MCUs. $\bullet$ A processor - ARM ${ }^{\circledR}$ Cortex ${ }^{\circledR}$ - M4 MCU with a client committed $256 \mathrm{~KB}$ of RAM and a discretionary $1 \mathrm{MB}$ of XIP streak. The ROM-based system incorporates an $802.11 \mathrm{~b} / \mathrm{g} / \mathrm{n}$ radio, baseband, and MAC with 
a capable motor for quick, secure web associations of 256-piece encryption.The framework incorporates inserted TCP/IP and TLS/SSL stacks, HTTP server, and various Internet conventions.

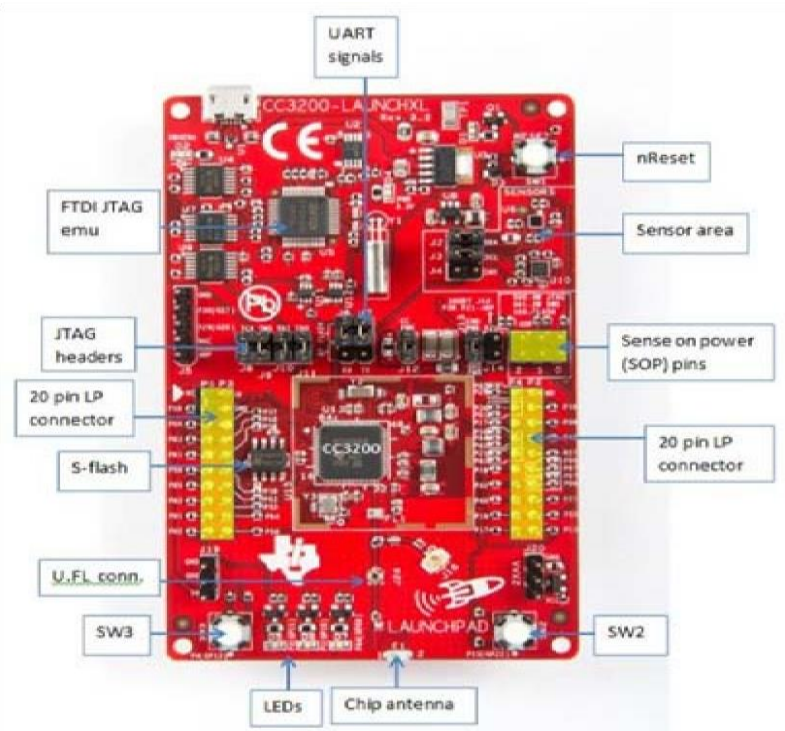

Fig. 1: TI CC3220S Hardware KIT.

The gadget underpins an assortment of Wi-Fi provisioning techniques including HTTP in view of AP mode, SmartConfig ${ }^{\text {TM }}$ Technology, and WPS2.0. The power-administration incorporates coordinated DC-DC converters that have an extensive variety of supply voltages. This subsystem empowers low-control utilization modes for expanded battery utilization The gadget includes a wide assortment of peripherals, such as camera interface, I2S, SD, UART, SPI, I $2 \mathrm{C}$, and 4-channel ADC.

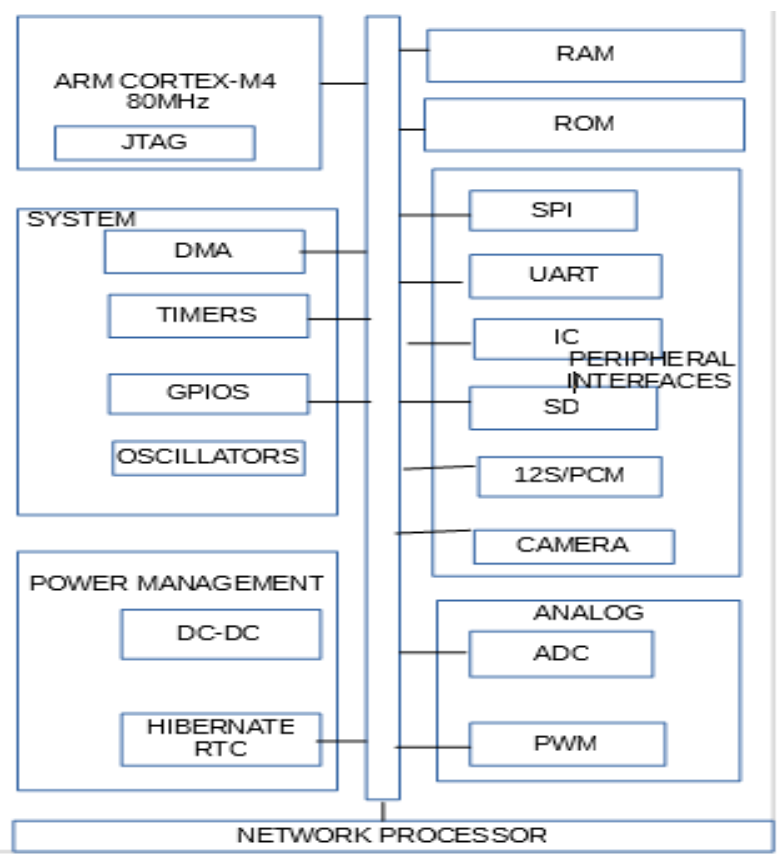

Fig. 2: TICC322OS Launchpad Overview.

\subsection{Camera - MT9D111}

MT9D111 is a 1/3.2 inch 2-megapixel CMOS Image Sensor with an integrated camera. It features a microcontroller and IFP with a real time JPEG encoder. It also includes a GPIO, which can be used to control external auto focus, optical zoom. The low light performance of MTD9111 is one of the best of digital clarity. It has power conserving properties such as low power standby mode and able to shutdown unused digital blocks.

\subsection{Temperature LM35 sensor}

The Temperature LM35 is a circuit sensor that can be used to measure temperature with an electrical output proportional to the temperature. It has an output voltage proportional to the Celsius temperature. It draws only 60 micro amps from its supply and possesses a low self-heating capability.

\subsection{LCD display}

Sharp LS013B4DN04 Memory LCD 1.3-inch screen of 96 x 96 pixels with Ultra-low-power consumption Provides excellent viewing angles and high-contrast images or text - Display is controlled serially using SPI

\subsection{Working model}

The Model can be enlightened by a case. Assume the administrator is supposing a visitor at his household, be that as it may, he isn't available there. Currently, by way of the guests grasp his household the administrator resolves to raise a video call. Be that as it may, nowadays the admin can crush digits additional than 1 , (meant for the sample, 3 for lights, 4 for the fan, etc) or even can modify the security outline. If the customer goes out and meanwhile if he receives video call the gadgets are to be turned off and are activated after the phone call.As the model is transferred process and it may not get interrupted as the microcontroller controls the process.

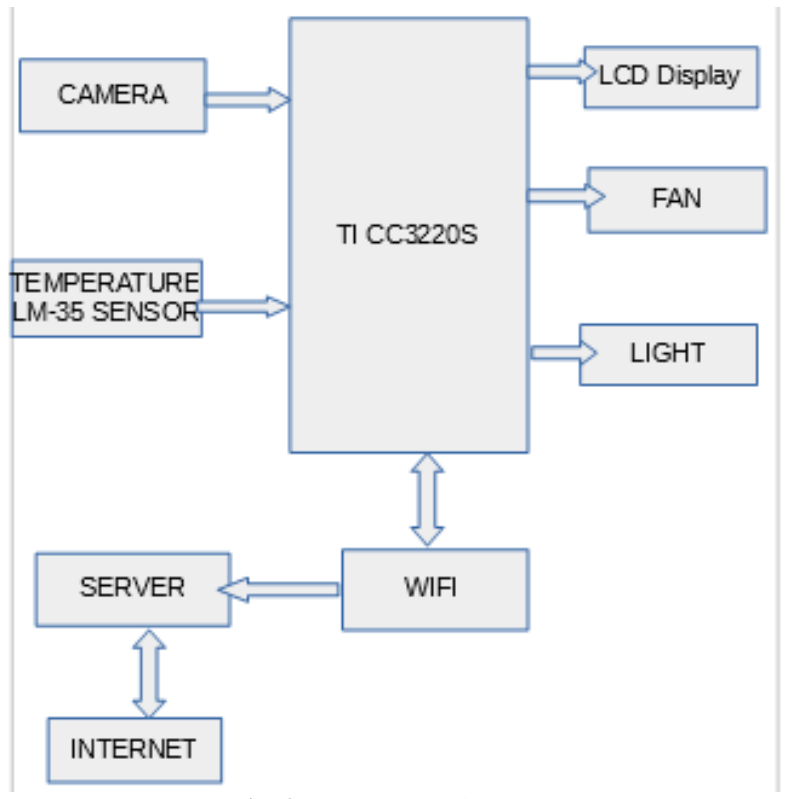

Fig. 3: Internal Architecture.

As the idea is to lay open to the customer's caution and to judge the scenario irrespective it is a visitor or a stalker going into his house, camera attached to the door side with the microcontroller will help the customer in making choices whether to activate the security outline or not. The caught snapshot of the visitor or stalker will be sent to the customer. The client can send the similar captured image to the police department if detects an intruder. Further, the security can be more enhanced where the customer can operate through mobile whether the home appliances are ON/OFF and desired to activate them anywhere before he reached home.

\section{Roposed design}

Temperature sensor and Camera are accessible by the doorsteps of a house. These sensors as famed beforehand describing the movement of persons. The person at doorsteps will be captured by the camera and send to the user. The user will be given an android mobile app software with a user id a password generated to it. As soon 
as user logs in the user get the information about the temperature, and whether the Fans and Lights are ON/OFF.

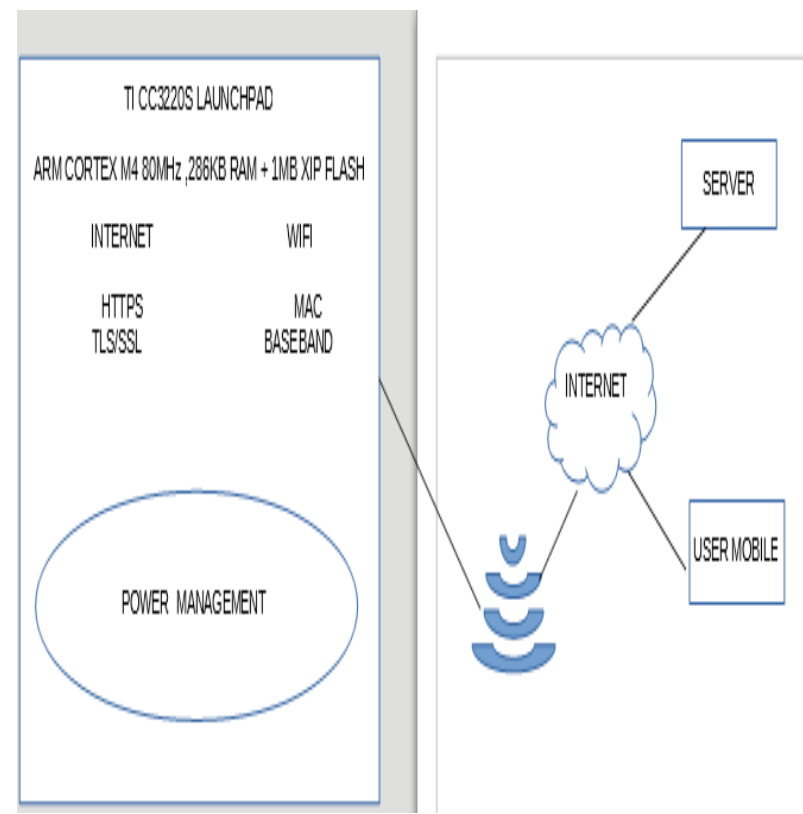

Fig. 4: Working Prototype.

the information about the temperature, and whether the Fans and Lights are ON/OFF. The captured image received by the user checks whether the intruder is known to him or not. If the intruder is known to the user he can enter the room and fans and light will be $\mathrm{ON}$ and access is generated by the user to switch on lights, fans. If the intruder is not known an alert message will be generated to the user and intruder will not be entered the room.

\section{Real time implementation}

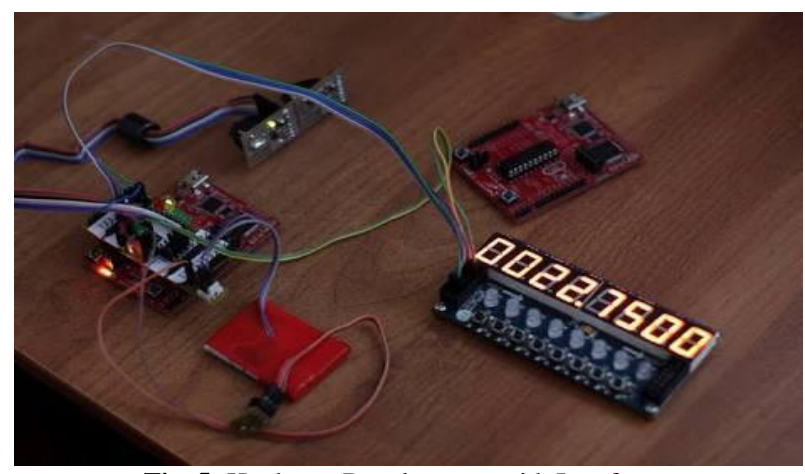

Fig. 5: Hardware Development with Interfaces.

The Launchpad framework when activated Temperature is set ON and show the temperature on LCD Display.If we test in heat and cooling temperature is varying according to environment parameters where you can detect oxygen levels in the home.

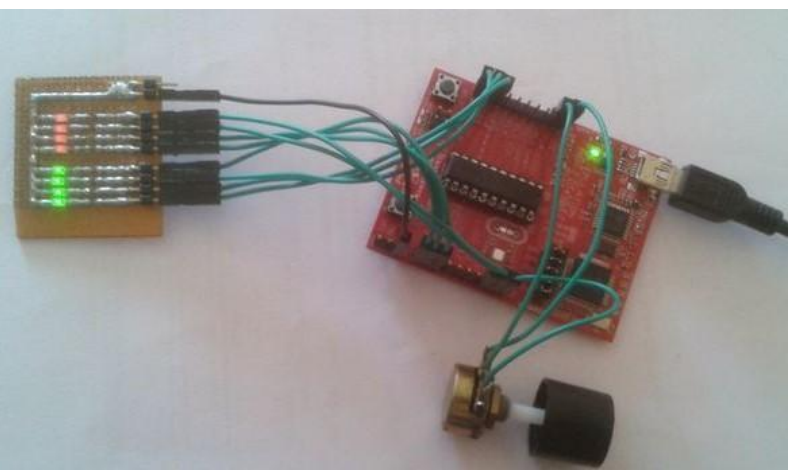

Fig. 6: Development of Hardware Kit with Final Prototype Peripherals.
The Launchpad framework automatically sets ON fan and light. FAN is tested mechanism by motor and Light is tested by LED. The user can ON/OFF the fan and light anywhere outside the home and by the time user gets in fan and light get $\mathrm{ON}$ which is set by the user by the mobile app.

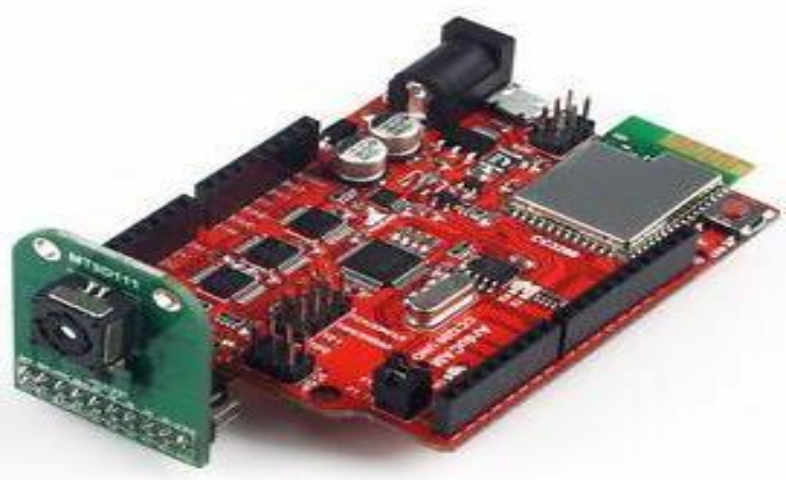

Fig. 7: Camera.

- The Launchpad framework sets the camera at doorsteps and capture the image of the visitor and send it to the user to detect the person is known to him or not.If not the user doesn't allow the person into the home

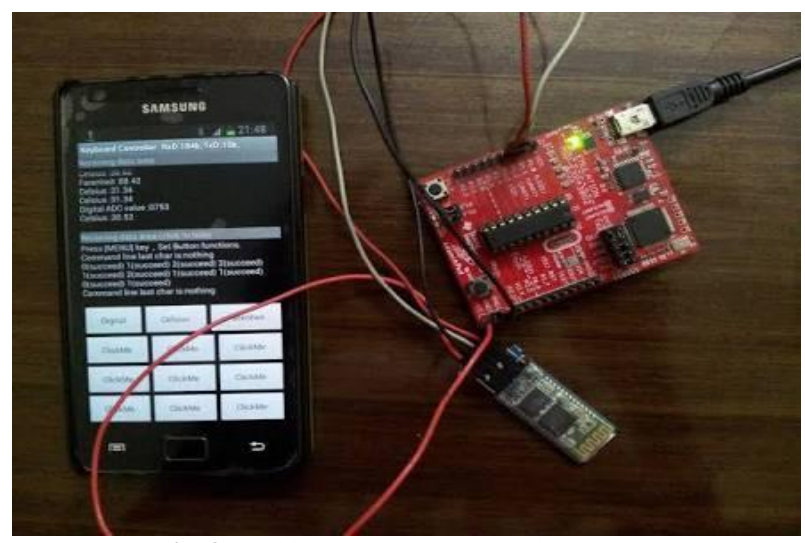

Fig. 8: Mobile App Control over the System.

The above Figures 5,6 and 7 as a whole are connected through a mobile app. Mobile App software is given to the user along with user ID and password the user logs in anywhere outside the home through which he can check the home applications are ON or OFF and gets the Fan, Light and other gadgets at home $\mathrm{ON}$ by the time he reaches home.

\section{Smart home system algorithm}

Algorithm

1) If motion sensed by the PIR sensor then

Turned ON Light

2) Else

Keep sensing

3) End if

4) If MQ5 gas value greater than or equals to 1050

then

Start Alarm

5) Else

Keep sensing

6) End if

7) If electromagnetic door sensor lost the line of sight connection for $30 \mathrm{sec}$ then

Start Alarm

8) Else

Keep checking

9) End if 
10) If temperature less than or equals to $24^{\circ} \mathrm{C}$ then

Turned OFF Fan

11) Else

12) If temperature greater than $24^{\circ} \mathrm{C}$ then

Turned ON Fan (Speed of Fan increased

with the increase in temperature)

13) End if

14) End if

\section{Conclusion}

In this paper, an IoT based algorithm is proposed for the smart home system to automate the Fan, monitor the gas leakage and notify by means of an alarm, intrusion detection and energy monitoring. The proposed algorithm was practically implemented on MSP435 based TI CC3220S L launchpad for the testing purpose. The result shows that the algorithm is capable to observe the motion of a human being, to observe the intrusion by monitoring and send the message to a mobile phone using GSM module. The temperature and power consumptions are monitored through a mobile App in globally and can be controlled being away from home. the system is efficient and cost-effective in terms of providing reliable information and automation. In future, this work can be to implement in a smart city to automate it as a smart home. to automate it as smart home.

\section{References}

[1] M. N. N. A. Asghar, M.H, "Principle application and vision in the internet of things (iot)," in Communication Technologies (GCCT), 2015 Global Conference.

[2] R.C. Y. O. K. Vithanage, C, "A comparison of the popular home automation technologies,"

[3] R. Pavithra, D., "Iot based monitoring and control system for home automation,"

[4] T. Ming Zhao, Chua, "Automatic face and gesture recognition, 2008. Fg '08. 8th IEEE international conference on,"

[5] Weimei Zhang," Study about IOT's application in Digital Agriculture construction", Electrical and Control Engineering (ICECE), 2011 International Conference, Yichang, IEEE, 2011, pp. 25782581.

[6] Takeshi Yashiro, Shinsuke Kobayashi, Noboru Koshizuka and Ken Sakamura,"An Internet of Things (IoT) Architecture for Embedded Appliances", Electrical and Control Engineering (ICECE), 2011 International Conference, Yichang, IEEE, 2011, pp. 2578-2581.

[7] Keertikumar M. J, Shubham M. and R.M. Banakar,'Evolution of loT in Smart Vehicles: An Overview", IEEE Computer Society, pp. 804-809.

[8] Takayuki Suyama, Yasue Kishino and Futoshi Naya"Abstracting IoT devices using virtual machine for wireless sensor nodes", Internet of Things (WF-IoT), 2014 IEEE World Forum, Seoul, 2014, pp. 367-368.

[9] Sean Dieter Tebje Kelly, Nagender Kumar Suryadevara, and Subhas Chandra Mukhopadhyay" Towards the Implementation of IoT for Environmental Condition Monitoring in Homes", IEEE SENSORS JOURNAL, OCTOBER 2013, VOL. 13, NO. 10, pp. 3846-3853.

[10] Shopan Dey, Ayon Roy "Home Automation Using Internet of Thing" 2016 IEEE.

[11] K.V.Daya Sagar, Jenny Martha K Asha," IMPROVING FAULT TOLERANCES WITH IN A CLOUD USING CROSSBAR NETWORKS", International Journal of Pure and Applied Mathematics, Volume 115 No. 8 2017, 65-70.

[12] K.V.Daya Sagar, Ch.RameshKumar,Ch.Sai Geethika, M.Yathisha, "International Journal of Pure and Applied Mathematics Volume 114 No. 10 2017, 319-327.

[13] K. V. Daya Sagar, Akella Pavan Kumar, Goli Sai Ankush, Thota Harika, Madireddy Saranya and Dasaraju Hemanth “ Implementation of IoT based Railway Calamity Avoidance System using Cloud Computing Technology", Indian Journal of Science and Technology, Vol 9(17), DOI: 10.17485/ijst/2016/v9i17/93020, May 2016. 